Revue d'histoire de l'Amérique française

ZAS REVUE D.HISTOIRE DE L'AMÉRIQUE FRANÇAISE

\title{
Le journal d'un militaire au nord-ouest canadien
}

\section{G. F. G. Stanley}

Volume 10, numéro 2, septembre 1956

URI : https://id.erudit.org/iderudit/301765ar

DOI : https://doi.org/10.7202/301765ar

Aller au sommaire du numéro

Éditeur(s)

Institut d'histoire de l'Amérique française

ISSN

0035-2357 (imprimé)

1492-1383 (numérique)

Découvrir la revue

Citer ce document

Stanley, G. F. G. (1956). Le journal d'un militaire au nord-ouest canadien. Revue d'histoire de l'Amérique française, 10(2), 263-278.

https://doi.org/10.7202/301765ar d'utilisation que vous pouvez consulter en ligne.

https://apropos.erudit.org/fr/usagers/politique-dutilisation/ 


\section{DOCUMENTS INEDITS}

\section{LE JOURNAL D'UN MILITAIRE AU NORD.OUEST CANADIEN}

$\mathrm{Au}$ printemps de l'année 1885, l'Ouest Canadien subit la seule insurrection sérieuse des métis et des sauvages de son histoire. $\mathrm{Au}$ fond, ce soulèvement ne fut qu'une protestation violente contre l'avènement inexorable de la colonisation; plus précisément ce fut le résultat de l'omission du gouvernement fédéral de régler les droits des Métis ou de résoudre les problèmes qui se produisirent lors de la disparition des bisons des grandes prairies. De juin 1884 à mars 1885 une agitation bruyante se continuait sous la direction de Louis Riel, agitation qui aboutit à la proclamation du gouvernement provisoire le 8 mars et à la victoire remportée par les partisans de Riel sur la gendarmerie et les volontaires du Prince Albert sous le commandement du Surintendant Crozier, au Lac aux Canards, le 26 mars.

Quatre jours avant l'escarmouche du Lac aux Canards, l'honorable Edgar Dewdney, le lieutenant-gouverneur des Territoires du Nord-Ouest, télégraphia au premier ministre, sir John-A. Macdonald à Ottawa, «Situation looks very serious. Think it imperative able military man should be on staff in event of militia going north.» ${ }^{1}$ Macdonald répondit: «General Middleton to proceed to Red River to-night. Order sent to Winnipeg Militia to be ready to move. ${ }^{2}$ C'est alors que le major-général Middleton, commandant en chef de la milice canadienne, partit pour le Nord-Ouest. Le 25 mars, le $90^{\mathrm{e}}$ bataillon de la milice de Winnipeg se réunit et se mit en route pour Qu'Appelle, la base principale des opérations. Dès que la nouvelle de l'échec du Lac aux Canards arriva à Ottawa, sir Adolphe Caron, le ministre de la milice, donna des ordres pour la mobilisation d'un corps expéditionnaire de 2000 hommes. Ce corps se composait des unités

\footnotetext{
${ }^{1}$ Archives publiques du Canada, Dewdney Papers, Vol. V; Dewdney to Macdonald, March 22, 1885.

2 Ibid., Macdonald to Dewdney, March 23, 1885.
} 
de la milice canadienne, parmi lesquels se trouvaient deux bataillons canadiens-français, le $65^{\mathrm{e}}$ de Montréal et le $9^{\mathrm{e}}$ de Québec. Le premier fut mobilisé le 28 mars et quitta Montréal quatre jours plus tard. Le 12 avril il arriva à Calgary avec le $92^{\mathrm{e}}$ de Winnipeg et la troupe de cavalerie du major Steele, tous sous le commandement du major-général T. B. Strange. Les troupes de Strange se rendirent à Edmonton et de là elles descendirent la rivière Saskatchewan du Nord pour surprendre l'ennemi. Le 28 mai elles infligèrent une défaite aux sauvages de Gros Ours à la Butte-aux-Français. Ensuite elles prirent part à la poursuite de Gros Ours.

Le $9^{\mathrm{e}}$ bataillon se mit sur un pied de guerre le 31 mars et partit de la ville de Québec le 2 avril. Il arriva à Calgary le 29 avril à neuf heures du matin. Il ne constitua pas une des unités de la brigade du général Strange. Son rôle était de protéger la population blanche du sud de l'Alberta et, ainsi, d'empêcher les sauvages de la ligue des Pieds Noirs de se joindre aux sauvages sous les armes dans la vallée de la Saskatchewan. Conséquemment le $9^{\mathrm{e}}$ régiment put fournir les hommes aux garnisons de Calgary, Langdon, Gleichen, Crowfoot et du fort McLeod. Ce rôle passif fut bien essentiel, même si l'excitation du combat et les coups de feu étaient absents. Après la reddition de Louis Riel et la défaite des sauvages à la Butte-aux-Français, la présence du $9^{\circ}$ bataillon ne fut plus nécessaire pour la sécurité de la contrée. On donna des ordres pour assurer le retour du régiment à Québec; mais avant leur départ, les soldats firent, aux frais du gouvernement, comme de simples touristes, un voyage dans les Montagnes Rocheuses. Le $9^{\text {e }}$ bataillon fut démobilisé le 27 juillet à la Salle d'Exercices de Québec.

Rares sont les chroniques officieuses des événements au Nord-Ouest du Canada pendant l'insurrection de Louis Riel; encore plus rares sont les récits des exploits militaires racontés par des membres des unités canadiennes-françaises. Les chroniques de Charles Daoust, George Beauregard et Oscar Pelletier ${ }^{3}$

3 Charles R. Daoust, Cent vingt jours de service actif, un récit historique de la campagne du $65^{e}$ au Nord-Ouest (Montréal, 1886); George Beauregard, Le ge Bataillon au Nord-Ouest (Québec, 1886); Colonel Oscar Pelletier, Mémoires (imprimé à cent exemplaires, Québec, 1940). 
sont les seuls ouvrages français qui décrivent ces faits historiques. Grâce au Révérend Père Adrien Pouliot, s.j., j’ai eu le privilège de consulter les manuscrits des journaux particuliers de deux militaires du $9^{\mathrm{e}}$ bataillon. Un de ces journaux, celui d'Arthur Potvin, ${ }^{4}$ est rendu public pour la première fois. ${ }^{5}$ D'une grande valeur historique, il complète le compte rendu de George Beauregard quant au rôle du bataillon dans les Territoires du NordOuest canadien; de plus, il révèle, d'une manière intime, la réaction et l'attitude d'un soldat volontaire soumis aux épreuves et à la rude discipline d'un corps militaire mal préparé et mobilisé à la hâte. Ce journal est une contribution à la fois intéressante et significative aux sources de l'histoire militaire du Canada français.

George F. G. StANLEY

Le Collège Militaire Royal du Canada.

\section{NOTES SUR L'EXPÉDITION DU 9e BATAILLON SE DIRIGEANT DANS LE NORD.OUEST}

Départ le 2 avril 1885.

Jeudi. On s'assemble au Drill Sher à $8 \mathrm{hrs.} \mathrm{P.M.} \mathrm{Après}$ avoir fait nos adieux à tous nos parents, amis et amies. A 9 hrs. P.M. tout le bataillon étant presque tout au complet, forma les rangs au milieu d'une grande foule rassemblée une dernière fois pour assister au départ de leurs amis ou parents. Un drapeau nous fut présenté en même temps par le peuple canadien français et Dr Chauveau. ${ }^{1}$ Après beaucoup de vivats de la part des Canadiens des clubs de Raquette qui voulurent bien nous faire l'honneur de venir nous reconduire avec flambeaux, nous défilâmes par les rues St. Louis, Buade, Fabrique, St. Jean, où la compagnie des Universitaires fut acclamée spécialement.

4 Arthur Potvin, l'auteur de ce journal, était étudiant en médecine quand l'insurrection éclata au Nord-Ouest du Canada en 1885. Il s'enrôla dans la $7^{\mathrm{e}}$ compagnie du $9^{\mathrm{e}}$ bataillon. Après sa démobilisation, il finit ses études, et pendant 57 ans le Dr Potvin exerça la médecine à la Basse-Ville de Québec. Il est décédé le 13 décembre 1945 à l'âge de 83 ans.

5 Je suis très reconnaissant à Madame Sylvio Caron de Québec pour sa gracieuse permission de reproduire le journal de son père.

1 Alexandre Chauveau, juge de sessions. M. Chauveau prononça un discours terminé par ces paroles peu rassurantes: «Si ce drapeau ne revient pas, c'est qu'il ne se trouvera personne pour le rapporter.» (R. Rumilly, Histoire de la province de Québec, V, 21-2.) 
On continua par les rues du Palais, St. Nicholas, enfin l'on arriva à la gare du Nord où la foule était tellement grande que le bataillon faillit se faire écraser. Après beaucoup d'adieux et d'encouragement de la part de nos amis et parents, les chars commencent à s'ébranler, un sifflent strident se fait entendre, et bientôt malgré notre bravoure on se sent mal à l'aise, car l'on quitte ... Québec et ... ! La soirée se passe gaiment, chansons, discours etc. Mais le plus malheureux, on ne pu dormir de toute la nuit.

Vendredi 3 avril. Arrivée à la gare St. Martin à 8 hrs. A.M. et malgré nos plaintes on ne pu déjeuner qu'à $91 / 2 \mathrm{hrs}$. A.M. Départ de St. Martin, on se change de train, et on embarque sur la ligne du pacifique. Nous n'arrêtons pas à Montréal malheureusement. Je suis malade mais après avoir eu des soins je reviens sur pieds. A 2 hrs. P.M. diner dans un hôtel aux frais du gouvernement et ceci à Calumet. Voici le menu: un œuf, un crocsignol, ${ }^{2}$ une sandwich et une tasse de café. C'était beaucoup pour des gens qui n'avaient pas mangé que du Corn Beef avec du pain sous le pouce. Waterloo pour Jolicœur. ${ }^{3}$ Nous arrivâmes à Ottawa à $6 \mathrm{hrs}$ du soir et là je ne pus desbarquer. Je ne vis que Victor Bélanger ... j'envoie des cartes de postes à Ottawa et à Québec. Nous demandons le souper. Il était $7 \mathrm{hrs} \mathrm{P.M.} \mathrm{On}$ nous répond qu'il fallait aller prendre le souper 28 milles plus haut, dans une place nommée Carleton. Hourrah! Nous mangeons tant que nous voulons, plus de rations, et dans un hôtel assez vaste pour contenir tout le bataillon «Hotel Carleton 》. Là j'ai mangé comme pas une maîtresse d'école. Là à Carleton nous couchons car nous venons d'apprendre que le $65^{\mathrm{e}}$ Bataillon de Montréal est bloqué à 50 milles plus haut qu'ici. Il fait un temps de chien. Nous ne pouvons dormir, cependant après avoir écrit jusqu'à $3 \mathrm{hrs}$ du matin, je puis dormir jusqu'à 6 hrs.

Samedi. Nous déjeunons à $9 \mathrm{hrs}$ au même hôtel à Carleton. Cette ville a une population de 4000 hab. et n'a que 3 habitants canadiens. Je suis allé avec d'autres visiter la manufacture de laine de Carleton dont le propriétaire est Wm. H. Wylie. Arrivée de notre chapelain M. Faguy. ${ }^{4}$ Visite de la ville. Je vais acheter du papier et on me fait présent d'une orange (c'était une jolie fille). Diner à $4 \mathrm{hrs}$ à la même place. Nous attendons le départ pour Winnipeg, enfin à 7 nous partons ... Belle réception à $\mathrm{Al}$ mond Station (village - 3000 hab.) à 15 milles de Carleton...

2 i.e. croquignol.

3 Philippe Jolicœur, caporal, $7^{\mathrm{e}}$ compagnie.

4 L'abbé François Faguy. 
Pakenham suivante de Almond. Aronbrior ${ }^{5}$ grands hourrahs. Pembrock ${ }^{6} 6$ à 7000 hab. 3000 Canadiens. Magnifique réception. Ici je vis Walsh qui a un frère à Winnipeg.

Dimanche. Pâques. Mattawa 580 milles de Québec. Nous arrivons dans cette espèce de ville le jour de Pâques, 5 avril 1885 , à $7 \mathrm{hrs}$. Déjeuner à $81 / 2$ hrs. C'est une ville de 1000 hab. dont les $2 / 3$ sont Canadiens. Cette place est encaissée dans les montagnes, ce qui lui donne une riante vue. Traversée par une belle petite rivière bordée de moulins, dont le nom est Keepway. Ici on a eu une parade officielle. Visite officielle du Chapelain Revd. Faguy, discours du Chapelain et hourrahs en son honneur. J'ai eu le plaisir de lui presser la patte. $10.40 \mathrm{hrs}$ A.M. passons à North Bay, place assez considérable situé sur le lac Nipissing, 80 milles de longueur. On soupe à Bisquittation ${ }^{7}$ dans une espèce de hutte. On n'avait que de l'orignal ou du cheval je crois, car c'était très dur. Pour dessert, de la compote. Là je rencontrai M. Sanders, frère de M. Sanders de Québec.

Lundi, 6. Parade officielle. Nous arrivons à une place à 62 milles du lieu où nous devons laisser les chars. ${ }^{8}$ Nous déjeunons avec des sandwichs, mais hélas, quelles sandwichs... pouah ! je ne puis en manger... diner semblable. Je grignotte quelque peu, ensuite on joue au bluff quelque peu, puis visite du chapelain. Il reste quelque temps avec nous. Nous venons de passer sur trois ponts immense, longs de 300 à 400 pieds et haut de 100 à 150 pieds. On a la chair de poule car nous sommes presque les premiers qui passons sur ces ponts. Presqu'à tous les milles, nous rencontrons un village, formé de huttes en bois ronds et haut de 4 à 5 pieds.

5.40 P.M. Dog Lake. Partis sur 50 voitures trainées par 2 chevaux chacune, la première étape fut de 30 milles. La nuit on peut dormir, débarquer, rembarquer.

Mardi, 7. Arrivée au petit village où l'on eut assez bon réveillon à $3 \mathrm{hrs}$ du matin. Tout le bataillon (presque) couché dans une chétive cabane sur le foin. Puis réveillé vers $4 \mathrm{hrs}$. du matin et repartis dans les mêmes voitures. Nous avions 16 milles encore. Un cheval de ma voiture meurt. Obligé de faire le reste à pied, c-à-d 7 milles. Il pleuvait depuis le départ.... Puis arrivé pour prendre le train. Il faut rester tous ensemble

5 Arnprior, Ont.

6 Pembroke, Ont.

7 Buscotasing, Ont.

8 Le chemin de fer Pacifique n'était pas complet à cette date-là. Il existait encore des interstices dans la ligne au nord du lac Supérieur. La voie ne fut livrée au trafic que le 6 juin 1886 . 
dans une tente toute trouée, l'eau dégorge sur nos têtes. Il est 2 hrs. P.M. et pas de manger !!! Enfin on nous donne un morceau de pain et un peu de corn beef. A 5.30 P.M. nous embarquons. Il pleut encore et il fait très froid. De plus il faut faire le voyage sur des chars plate-forme, cependant on ne dit mot... Quel nuit nous passâmes; nous étions 62 soldats dans le même char, la pluie nous inondait. A chaque moment l'on pensait voir dérailler les chars, car la ligne est très mauvaise sur ce bout. A minuit n'en pouvant tenir, l'on ordonne au conducteur d'arrêter les chars et l'on députe un soldat vers les officiers qui étaient tous dans un bon char, ayant un poële etc. Les soldats se lamentaient, tout le monde pensait en mourir, moi-même, il me prenait des envies de me jeter en bas des chars, et beaucoup furent sur le point de faire la même chose, tant le froid était intense. On demande des hommes pour aller chercher des couvertes. Dispute avec le lieutenant Fiset, ${ }^{9}$ il n'en faillait pas plus pour me faire passer devant une cour martiale. M'ayant enveloppé de couvertes, je souffris moins, mais hélas, je ne pus dormir et le matin n'arrive plus.

Enfin après bien des souffrances $5 \mathrm{hrs}$. arrivent, et joyeux, nous saluons le retour du jour. Moi-même j'avais les pieds gélés, surtout un talon. Au milieu de nos souffrances du froid j'entends une voix qui s'écrie d'un air découragé, «Moi, je ne reviens plus ». Je me retourne, car c'était mon voisin... et j'aperçois Alain, ${ }^{10}$ mon compagnon, car c'était lui qui avait lâché ce bon mot. Nous avons fait 110 mil. ainsi.

Mercredi, 8 av. Nous allâmes tous déjeuner, car nous avions besoin de refaire nos forces. C'était à Iron Bay. J'allai me rechauffer. Après 2 heures nous nous embarquons dans le chars pour faire 15 milles. Ce chemin fait, on débarque et avec tout notre bagage sur le dos, nous faisons un mille et nous allons nous établir dans une barque où nous couchâmes tous dans la cale d'une barque dont le nom est «M. L. Breck of Kingston ». Elle était amenée dans une baie du Lac Supérieur, appelée Port Munroe. On alla souper à $4 \mathrm{hrs}$. dans les tentes, et ce soir-là tout le monde dormit, mais moi me trouvant près de la porte de la cale, toutes les sentinelles me passèrent sur la tête en allant faire leurs garde. Le lever à 5 hrs. A.M.

Jeudi, 9 avril. Déjeuner $5.30 \mathrm{hrs}$. et parade officielle à $6 \mathrm{hrs}$. Ensuite l'on part à pieds pour faire une marche de 28 milles. Pour moi je marchai quelque peu et j'embarquai dans

9 Lieutenant Elzéar Fiset, $4^{\mathrm{e}}$ compagnie.

${ }_{10}$ Ludger Alain, $7^{\mathrm{e}}$ compagnie. 
une des voitures qui se trouvaient à côté de nous pour les malades. On fit le trajet en 6 et demie. Arrivé à Nicholas Port, on reprit les chars découverts pour faire 15 milles, c'est à dire jusqu'à Jack Fish Bay, une des nombreuses baies du lac Supérieur. J'avais oublié de dire que nous avions fait notre marche de 28 milles continuellement sur le lac Supérieur sur la glace.

Arrivée à Jack Fish Bay. On s'arrima dans une espèce de hangar pour y passer la nuit et nous allâmes prendre le souper et le diner, car nous avions déjeuné le matin à $5.30 \mathrm{hrs}$. Je mangeai 2 grandes tranchés de gros lard. Après le souper j'allai me coucher dans un petit grenier du hangar et je dormis assez bien.

Vendredi, 10. Lever à $6 \mathrm{hrs}$. Presque tous les soldats et officiers ont attrapé des coups de soleil. Temps malsain et humide. Il neige et cette neige mouille. Nous prenons le déjeuner à $8 \mathrm{hrs}$. Même nourriture que la veille, car nous sommes encore à Jack Fish Bay. On dit que le lac Supérieur a 400 milles de long, sur 200 de large. Tous habillés, nous attendons le signal du départ. Cependant on vient nous dire que nous ne pouvions partir qu'à 4 hrs. P.M. On déshabille, quelques uns dorment et d'autres jouent aux cartes. On s'embarque à 5 hrs. P.M. 12 voitures. Nous sommes tous malades dans le trajet; on nous avait presque empoisonnés avec leur lard de chantier. Il y en eut jusqu'à 5 par voiture qui furent obligé de se mettre en position ...

Samedi, 11 a. Après avoir fait nos 24 milles, nous débarquons à 12.30 après minuit, et nous fûmes obligés d'attendre pendant 4 longues heures autour du feu. Il neigeait... a ah quelle vie ! Plusieurs se vendaient au diable et vouaient les officiers du $9^{\mathrm{e}}$ à tous les diables; cependant ils étaient aussi à plaindre que nous. Tous les soldats sont encore embarqués pèle-mêle dans les chars plate-forme, sans bancs. Les soldats ne savent où se mettrent. Ils tombent de sommeil. Après avoir fait 10 milles ainsi, on va prendre nos quartiers dans un steamer « The Georgian of Kingston ». Alain et moi, nous allons prendre une bonne tasse de thé dans une maison de pension et nous revenons dormir jusqu'à 7 hrs. On embarque immédiatement sur les chars et nous faisons 50 milles jusqu'à Mackay's Land [ing] et là encore nous avons un portage de 12 milles. Quelle misère nous avons eu. Il faisait très froid sur le lac Supérieur, mais cependant nous avions l'espoir de nous reposer à Red Rock où nous devions prendre les chars pour se rendre à Port Arthur et de là à Winnipeg sans changer de char. Nous avons tous des premières classes. 
Maintenant quelques mots sur les mœurs et les manières de vivre des divers bourgades que nous avons passé sur notre chemin. Ce sont tous presque des tentes pour abriter les travaillants sur le Pacific. Ils vivent de lard et de beans, mets que je n'en jamais voulu gouter. Les provisions se vendent très cher. Une mauvaise bouteille de whisky se vend à $\$ 5$; encore est-il très difficile de s'en procurer car la loi Scott ${ }^{11}$ est en vigueur partout, excepté à partir de Port Arthur.

Aujourd'hui nous avons passé sous 2 magnifiques tunnels de 300 à 200 pds. de long. Les entrepreneurs ont $\$ 165$ par pieds, c-a-d jusqu'à la hauteur. Tout le monde est joyeux, c'està-dire notre compagnie, quant aux autres... Arrivés à Port Arthur à 9.30 P.M. Nous prenons un bon souper et diner dans un hôtel de cette ville. Ville assez grande, de 7 à 8 milles hab. avec de magnifiques magasins, grandes rues. Assez belle réception. Nous restons là une heure et demi à peu près et nous donnons l'hospitalité dans nos chars à 4 soldats, habits rouges du $12^{\text {e }}$ Bataillon de Toronto. ${ }^{12}$ Ensuite tout le monde se couche et je dormis jusqu'à $10 \mathrm{hrs}$. le lendemain, était dimanche.

Dimanche, 12. Lever à $10 \mathrm{hrs}$. A.M. avec gros rhume. Les bruits courent que nous arrêtons pas à Winnipeg et que nous continuons jusqu'au fort McCloud ${ }^{13}$ aux pieds des Montagnes Rocheuses; c'est ce que nous allons voir dans quelques heures. Après avoir crié quelques temps après le déjeuner, on nous le donne à 11.30 A.M. Ensuite à 2 hrs. P.M. nous débarquons au Portage du Rat ${ }^{14}$ où nous prenons un second repas dans un hôtel; là la bière nous fut donné à satiété. Ensuite l'on reprend les chars pour se rendre à Winnipeg. Nous avions encore 146 milles à faire. Enfin à $71 / 2 \mathrm{hrs}$. P.M. nous arrivons à Winnipeg où nous allons prendre nos quartiers en dehors de la ville dans des tentes préparées d'avance et que le $65^{\mathrm{e}}$ de Montréal venait de quitter pour se rendre aux frontières. Pour la première nuit que nous passons sous des tentes, il fait très froid. Je dormis assez bien cette nuit-là.

11 Le Canada Temperance Act (appelé la loi Scott d'après son promoteur au parlement du Canada, Sir Richard W. Scott), adopté en 1878, permettait à chaque comté de chaque province de déterminer, par vote populaire, la permission de faire usage de spiritueux ou de les prohiber.

12 L'auteur voulait dire, probablement, le $10^{\mathrm{e}}$ bataillon « Royal Grenadier » de Toronto qui avait précédé le $9^{\mathrm{e}}$ au Nord-Ouest du Canada. Le $10^{\mathrm{e}}$ bataillon était arrivé à Port-Arthur le 7 avril. (T. E. Champion, History of the 10th Royals (Toronto, 1896), 125).

13 Fort McLeod situé au bord de la rivière Old Man au sud de l'Alberta.

14 Aujourd'hui connu sous le nom de Kenora. 
Lundi, 13. Lever 5.30. Déjeuner $9 \mathrm{hrs}$. Je visite la ville avec plusieurs autres. Nous visitons les principaux établissements et surtout le Parlement. Diner 3.00. Sortons encore en ville. Diner $7 \mathrm{hrs}$. Nous sortons en ville jusqu'à $10 \mathrm{hrs}$. Je suis allé à St. Boniface où j'achetai une paire de souliers sauvages jaunes. Tous des canadiens à St. Boniface. Il y a un couvent, un collège, et un hôpital. La rivière Rouge sépare Winnipeg de St. Boniface. La rivière Saskatchewan ${ }^{15}$ est à 2 arpents de notre camp. Coucher à $11 \mathrm{hrs}$. Je n'ai pas dormi de la nuit, car il fait trop froid. Nous ne sommes que 4 dans notre tente, car tous les autres sont chauds et dans la ville.

Mardi, 14 avril. Lever à 5.30. Déjeuner et ensuite parade. Dans l'avant midi nous faisons prendre notre portrait 4 fois. Nous avons le diner, puis parade à $2 \mathrm{hrs}$. et à $4 \mathrm{hrs}$. Il nous fut très difficile de se procurer une passe du Colonel; nous l'eûmes enfin à 5 hrs. et Alain et moi nous allâmes à la Young Men's Christian Association Rooms. Nous assistâmes à un meeting où le ministre explique la bible etc. J'achetai des œufs ce jour-là pour mon déjeuner et je dinai avec du lait. Beau temps toute la journée.

Mercredi, 15. Routine jusqu'à midi, et puis l'après-midi nous avons une parade à 2 heures, où nous pratiquons l'escarmouche pendant environ une heure. Après la parade, De Guise et moi, nous avons une passe du Capt. et du Colonel de 5 hrs. P.M. à 11 hrs. P.M. Nous soupons chez Jacques Bonneau, allons au théâtre où Olivette fut joué. Revenu au camp. Je dors quelque peu et le lendemain le Colonel Amyot, le Lieut.-Col. Roy, une partie des officiers étaient venu me voir. Je pars pour l'hôpital en carosse. Je fus 2 jours sans manger à l'hôpital. Je pris un peu de lait et je ne pus le garder. J'avais une attaque d'inflammation d'intestins. La première nuit, 2 médecins restèrent auprès de moi avec plusieurs autres. On pensait que j'allais mourir. Je les entendais parler. La nourrice était effrayée mais moi je ne pouvais remuer. Quelques fois les crampes me prenaient, alors on était obligé de me tenir, car je me serais jeté à bas de mon lit. Nous sommes assez bien à cette hôpital. Ce que je trouvais de ridicule, c'est que tout malade que j'étais on m'obligea de me coucher, avant de monter dans la chambre où était mon lit. Là je perdis ma montre et ma pipe. Je restai à l'hôpital jusqu'à 5.30 P.M. de samedi 18 avril 1885. Nous étions 7 soldats du $9^{\circ}$ Bataillon. A. L. Laurencelle, Télesphore Trudel, DeGuise, Blais, Lortie, Miller, Bastien et moi.

15 M. Potvin fait ici erreur. La rivière se nommait l'Assiniboine. 
Je connus un jeune homme de Toronto, un homme D. H. McKay, qui me donna une commission pour le révd. Père Rédemptoriste Hayden, de l'Eglise St. Patrice de Québec. Je sortis de l'hôpital pas assez rétablis, et nous allâmes, Laurencelle et moi, prendre notre souper chez un Monsieur Laurendeau, Main Str. canadien, où nous fûmes très bien servis. Là je rencontrai un homme de la Basse Ville, un Monsieur Chaumette, d'autre fois à la station de feu, numéro 5 .

Samedi, 18 avril. Le camp change de place car l'eau montant à cause de la pluie avait envahi le camp, et à leur reveil les soldats étaient presqu'inondés. Maintenant il se trouve au bout de la rue Queen's. Je couchai chez Laurendeau, et le matin j'étais assez bien, cependant j'avais toujours le rhume.

Dimanche, 19 avril. Lever à 8. Encore chez M. Laurendeau. Je pris le déjeuner à 10.30 A.M. et je m'en retournai au camp où je fus très bien reçu par mes compagnons, eux qui me pensaient presque mort. Je changeai de tente et le caporal Paradis m'arrangea une place dans sa tente, par conséquent je changeai de compagnons de nuit: c'étaient Jules Paradis, Jolicœur, Henri Roy, Godin, Gaumond, Lavoie, Collet, et le petit Pion-Pion, Charles Dénéchaud. Le dimanche nous nous amusâmes assez bien, à nos tentes d'abord, où plusieurs citoyens de Winnipeg se trouvaient, Ms. Prince, Bonneau, Collet et d'autres encores. Le Capt. et lieutenant étaient avec nous. A $4 \mathrm{hrs}$. P.M. nous allâmes en ville où nous soupâmes presque tout le bataillon chez notre ami Laurendeau. Nous revenons au camp à 10 hrs. P.M. Nous avions passé une partie de la soirée avec nos amis de Québec, les soldats de l'école de carabine, ${ }^{16}$ qui étaient arrivés du matin. Eux aussi eurent beaucoup souffert sur le lac Supérieur. Il pleut toujours et il nous faut coucher sous nos tentes.

Lundi, 20 avril. On a une drille à 6 hrs. A.M. Nous ne pouvons avoir notre déjeuner qu'à $9.30 \mathrm{hrs}$. A.M. Encore nous n'avons qu'un peu de café, une demi-once de fromage et du pain et on nourrit les soldats qui travaillent comme des chiens avec si peu de nourritures ! Le diner un peu de soupe et de viande qu'on ne peut avaler ! Ouf que c'est beau d'être soldat!!! A $4 \mathrm{hrs}$. nous sortons, même routine. Il pleut toujours. J'avais oublié de dire qu'on avait eu une drille mémorable, pendant $2 \mathrm{hrs}$. sans interruption. Tous les soldats murmuraient dans les rangs.

16 Ce corps était le premier corps permanent d'infanterie du Canada confédéré. Il fut établi en 1833 et fut composé de trois compagnies, une compagnie chacune à Fredericton, St. Jean et Toronto. (G. F. G. Stanley, Canada's Soldiers (Toronto, 1954) 248). 
Mardi, 21 avril. Même routine. Il pleut encore. Nous drillons le matin et l'après-midi nous ne drillons qu'une heure, car la pluie est trop forte. Nous sortons et nous allons prendre le souper chez Laurendeau. Cette nuit-là fut très orageuse; l'eau entre dans les tentes pendant la nuit. Je pensais à tout moment que la tente ne tombait. On avait murmuré que le colonel a fait des démarches et nous changeons de camp pour la $3^{\mathrm{e}}$ fois. On veut avancer plus loin aussi, mais le ministre de la Milice (Caron) ne chante pas ainsi, parait-il. Ceux qui ont fait la garde cette nuit ont eu beaucoup de misères et ceux qui la feront aujourd'hui en auront autant.

Mercredi, 22 avril. En m'éveillant, on m'appelle pour faire la garde. Je m'exempte encore, car je crache les poumons depuis 8 jours et je ne peut me mettre dans l'eau pour 2 heures de suite sans mourir certainement. Nous dinons, puis nous changeons de camp. Nous allons dans un ancien hôtel vis-à-vis l'hôtel Canada. ${ }^{17}$ Nous sommes à l'abri mais les côtés nous font mal le lendemain matin.

Jeudi, 23 avril. Lever à 5.30. Pas de parade le matin. Je sors pour aller porter les rations aux gardes de l'autre camp. En arrivant on m'annonce le départ pour Swift Current à $6 \mathrm{hrs}$. P.M. Nous ne pouvons sortir qu'en usant de ruse, moi et Jules Paradis. On soupa en dehors et j'acheta 2 portraits de la compagnie No. 7 que j'envoyai l'un chez nous et l'autre à Damase. A six heures nous partons en chantant (bataille du Capt. Drolet et du Capt. Garneau avec 3 Anglais, bon coup de poing du sergent LeClerc) ${ }^{18}$ On embarque sur les chars du Pacifique encore; puis la Compagnie de la Baie d'Hudson nous fait donner chacun $11 / 2$ lbs de tabac. Après avoir pressé la main de plusieurs de nos amis de Winnipeg, on file. On arrête quelques instants à 60 milles de Winnipeg, au Portage de la Prairie. Il était $71 / 2 \mathrm{hrs}$. P.M.

Vendredi, 24 avril. Le premier arrêt est à Qu'Apelle à 500 milles de Winnipeg. ${ }^{19}$ Ensuite vient Regina. Là nous ne pûmes

17 Il s'appelait le Board of Trade Hotel.

18 George Beauregard op. cit. 26, racontait cet épisode comme suit: «A notre départ de l'hôtel, un individu bien mis, ayant assez l'apparence d'un homme bien élevé, probablement le sang échauffé parce qu'il n'avait pas mis d'eau dans son vin, s'approcha de nous et commenca à nous insulter. C'était un anglais ennemi du nom canadien-français. Il a voulu porter l'enthousiasme jusqu'à frapper un de nos officiers. Mais on l'a prévenu, un sergent lui a appliqué un coup de poing dont il se souviendra longtemps. Voila ce qu'il a dû appeler, dans sa langue maternelle, short and sweet.» 19 Une compagnie du 57e de Peterborough fit partie du « Midland Regiment », régiment ad hoc de l'Ontario central. 
avoir de déjeuner. On se rend à Moose Jaw, où l'on se donne le plaisir de prendre une bouchée; il était $11 \mathrm{hrs}$. A.M. Après le déjeuner nous prîmes une partie de plaisir avec les Sioux, sauvages qui résident à 3 milles en arrière de Moose Jaw. Beaucoup du bataillon achetèrent des tomahawks ou d'autres choses appartenant aux sauvages ou squaws. Je n'ai jamais vu de sauvages aussi laids. Ils étaient tout pour la plupart tatoués et peints en guerre. Nous restâmes à Moose Jaw $11 / 2$ hrs. On embarque et nous filons dans la direction de Swift Current où nous arrivons à $61 / 2 \mathrm{hrs}$. P.M. Il y avait déjà un bataillon de camper, le $91^{\mathrm{e}}$ de Winnipeg. Nous avions très faim. Cependant il nous fallut attendre encore $21 / 2 \mathrm{hrs}$. sous la pluie avant de dresser nos tentes, et nous n'eûmes pas de souper. Par hasard il nous restait quelques miettes de biscuit dans le fond de nos sacs à pain. On nous donna trois couvertes chaque homme, car nous étions obligés de coucher sur la terre humide, pas de foin! Je dormis très bien cette nuit-là.

Samedi, 25 avril. Lever à 5.30 et parade jusqu'à 7.30 . Il avait neige la nuit et il faisait très froid le lendemain. Nous avions de la difficulté même à tenir nos carabines! Nous prenons le déjeuner, toujours avec du biscuit de matelot trempé dans du café ou du thé, car nous ne pouvons pas les distinguer par le gout. Par chance il était un peu chaud. Seconde parade à 10 hrs. en brigade, jusqu'à midi. J'avais un rhume épouvantable depuis 15 jours. A la visite du docteur ce matin je me fis exempte de la parade et je pense m'en exempter pour plusieurs jours.

Un mot sur Swift Current. C'est un petit village de 4 maisons parmi lesquelles est l'entrepôt de la Compagnie de la Baie d'Hudson. Il y a aussi plusieurs huttes de sauvages. Le colonel, dans ses ordres du jour ce matin, défend de leur parler ou de trafiquer avec eux. Mon ami Alain vient d'être reçu trompette ce matin et c'est pour le reste de la campagne. La garde est très sévère et la discipline est soignée de près, car la nuit dernière dans l'autre camp à côté de nous, ils eurent deux chevaux de voler par les métis. Hier au soir on disait que le $9^{\mathrm{e}}$ bataillon devait aller camper plus haut que Swift Current à 25 milles sur les bords de la Saskatchewan. Parade à 2 hrs. P.M. Nous n'eûmes jamais de si belles parades. Nous avons pratiqué l'escarmouching, et nous avons marché pendant cette parade de la valeur de 6 milles dans les plaines. La parade dura $4 \mathrm{hrs}$. Le soir nous soupâmes encore avec deux biscuits et du thé. Voici le menu de nos repas à Swift Current. Le matin: 2 biscuits et une tasse de thé avec une demi once de fromage. Le Midi: 2 biscuits et de la viande en boite $1 / 2 \mathrm{lbs}$. Le soir: même chose que le matin. 
Nos genoux nous servent de table et nos chaises sont la plaine humide.

Dimanche, 26 avril. Lever à $7 \mathrm{hrs}$. Parade et messe à 9 hrs. Rien de plus imposant et de plus solennel; à l'élévation nous présentâmes les armes. On chantait des chants sacrés. Vraiment on se sent remué jusqu'à dans le profond du cœur. Après la messe le colonel nous avertit de nous tenir prêts à partir le soir même pour Calgary, nouvelle que l'on reçut avec des hourrahs frénétiques.

Lundi. Rien de remarquable.

Mardi. A notre départ, qui s'effectua vers les $5 \mathrm{hrs}$. P.M., les soldats du $57^{\mathrm{e}}{ }^{19}$ et les Midlands vinrent nous reconduire, tambour battant. C'était magnifique. A 7 hrs. P.M. nous passâmes à Maple Creek où nous vîmes des Canadiens et 25 hommes de la police à cheval, parmis lesquels se trouvent un nommé Levesque de Québec, dont la mère demeure sur la rue St. Paul, Palais. Nous passâmes encore une nuit sans dormir, mais que nous importait, nous avancions ...

Mercredi, 29. A 5 hrs. A.M. nous apercevons au nord-ouest les Montagnes Rocheuses recouvertes de neiges. On arrive à Calgarry à $7 \mathrm{hrs}$. Peu après notre arrivée nous allons souper, déjeuner et diner dans un hôtel; mais il faut remarquer que nous fîmes ces trois repas dans un seul. Ensuite nous montâmes les tentes et je fus assez heureux de pouvoir voler un peu de foin. Le reste de la journée nous n'eûmes pas de parade. Le soir nous allâmes visiter la ville de Calgarry. C'est assez joli et il y a de très beaux magasins. Il n'y a que 13 mois et Calgarry ne comptait que 2 maisons et encore étaient-elles petites.

Jeudi, 30. Le matin le colonel nous donne la lecture d'une dépêche qu'il venait de recevoir. Elle portait que la bataillon arrivant ici devait envoyer 2 de ses compagnies au fort McLeod à 100 milles de Calgarry, aux pieds des Montagnes Rocheuses; les comp. Nos. 1, 2, 8 furent envoyées en avant. Nous nous séparâmes avec regret de nos joyeux compagnons de la $8^{\mathrm{e}}$, car elle est composée d'universitaires.

Le lendemain de la journée se passa comme d'habitude. La nourriture est comme à Swift Current, le biscuit de matelot est à l'ordre du jour. Ils s'en servent même pour faire des soupes aux biscuits. Ce jour-là je fus de garde. Pendant mon garde de 2 hrs. à 4 du matin, je vis un grand troupeau de chevaux qui viennent s'abreuver à la rivière Elbow à 2 arpents du camp.

Vendredi, 1 mai. Pas de drill de la journée. C'est le départ de nos amis. Le matin nous avions eu la messe. Le reste de la journée se passe comme d'habitude. 
Samedi, 2 mai. Toute cette journée ressemble aux autres.

Dimanche 3 mai. Parade de la messe à 9 hrs. A.M. Le col. Evanturel ${ }^{20}$ insulte un servent (Chabot) ${ }^{21}$ et Collet ${ }^{22}$ est arrêté par le sergent-major. La messe fut dit par le Révd. Père Claude de la mission. Il fit une courte allocution après la messe. Je me rendis aux vêpres le Dimanche à la Mission. Après les vêpres nous allâmes avec un Canadien de Joliette dans sa chambre, puis nous nous fîmes donner des renseignements sur Calgarry. On apprend la mort de Marois. ${ }^{23}$

Lundi, 4 mai. Notre compagnon Collet s'arrange très bien son affaire. Cependant si cette affaire avait causé une émeute, il se faisait fusiller. L'après-midi nous recevons une lettre du Père Lacombe, ${ }^{24}$ qui nous donne des conseils concernant les sauvages et de plus de nous tenir sur nos gardes, car, malgré leur bonne foi dans le temps présent, un beau discours peut les soulever contre nous dans $2 \mathrm{hrs}$. Puis il termine en disant que les Pieds Noirs se disposaient à danser selon leur manière près de notre camp, et cela pour montrer qu'ils sont en bonne intelligence avec nous et qu'ils veulent la paix. Leur grand chef, Stomic Otacon, ${ }^{25}$ était à leur tête avec 2 sorciers. Ils avaient une tambourine sur laquelle frappaient sans interruption 4 sauvages. Ils dansèrent ainsi toute l'après-midi. Après leur danse, le colonel leur fit distribuer du biscuit, du tabac, du thé et de la viande. Puis nous fîmes en leur présence l'exercice du sabre. Puis nous revînmes au camp.

Vendredi, 9 mai. Je finis ma garde à $101 / 2$. Je ne pus dormir de la journée. On nous averti qu'un souper et une soirée de music et de chants nous est donnée par les dames de Calgarry. Alors nous commençons à nous brosser et laver. L'aprèsmidi le capitaine nous donne la paye de 10 jours. Puis après le souper au camp, nous nous mettons en rang, puis nous allons nous amuser avec les jeunes filles de Calgarry. Nous arrivons dans une grande salle de 300 pieds sur 50 . Il y avait déjà plusieurs jeunes filles de rendus. Puis les tables étaient abondam-

20 Lieutenant-Colonel Arthur Evanturel.

21 Le sergent Joseph E. Chabot, 7e compagnie.

22 C. A. Arthur Collet, soldat, $7^{\mathrm{e}}$ compagnie.

23 Théophile Marois, soldat, $4^{\mathrm{e}}$ compagnie. Marois tomba malade pendant que le bataillon faisait son séjour à Swift Current le 24-28 avril. Il fut transporté à l'hôpital où il mourut plusieurs jours plus tard. Un autre soldat, Achille Blais, de la 7 e compagnie, est mort le 30 avril à Winnipeg. 24 Le R. P. Albert Lacombe, O.M.I., grand missionnaire chez les Cris et chez les Pieds Noirs. Les sauvages l'ont surnommé Homme au bon cœur. 25 Tête de Bœuf. 
ment, on est tout étonké de voir de si bonnes choses, nous qui ne mangeons que du fromage et du pain. Après le discours du maire, le colonel Amyot ${ }^{26}$ fait un joli et charmant discours, dans lequel il louange surtout les femmes de Calgarry. Puis vient celui d'Evanturel. Ensuite l'on reçoit l'ordre de s'approcher des tables. Je n'ai pas besoin de dire que je fis honneur au souper. Après le souper, les chansons et la music se continuèrent. Nous ne fûmes jamais si bien traités et reçus, si ce n'est à Québec. Nous laissâmes la salle à 11.30 hrs. P.M. J'étais content de dormir, moi qui n'avais dormi la veille. A $1 \mathrm{hrs}$. du matin le camp est tout éveillé par le cri «Le camp est attaqué, il y a des hommes cachés dans les billots. » Dans 5 minutes tout le monde est sur pied et prêt à combattre. Ils nous donnèrent des cartouches. Mais ce ne fut rien. Après bien de recherches nous ne trouvâmes rien; ils croyèrent trouver le camp endormi, mais le Canadien veille toujours. Puis nous nous recouchâmes tranquillement. Le lendemain, pas de drille, car il se trouve un pouce de neige sur la terre.

Samedi, 10 mai. La parade de 61/2 n'a pas lieu. Seulement à $10 \mathrm{hrs}$. nous paradons jusqu'à $111 / 2 \mathrm{hrs}$. L'après-midi nous allons tirer à la cible. Cette fois-ci je fis 2 points. A Swift Current je n'avais pas fait un seul point. Puis je vais en ville avec quelques autres. Le reste de la journée se passa comme d'habitude.

Dimanche, 11 mai. Lever à 5 hrs. Puis nous avons la messe à $7 \mathrm{hrs}$. dite par le missionnaire Lacombe. Après la messe, il nous fit un petit sermon sur l'évangile du jour « demandez et vous recevrez ». Après la messe nous formons un club de Baseball. Après le diner j'allai faire une visite au presbytère avec quelques autres, et à $4 \mathrm{hrs}$. P.M. nous entendons les vêpres. Puis nous retournons au camp après les vêpres. Après le souper nous jouons au Base-ball. Le soir je fus très malade du mal de dents. La rumeur qui circulait depuis 2 ou 3 jours, que les Comp. 3 et 4 devaient partir pour Gleichen, Crowfoot et Langdon, est confirmée, et elles partiront demain sous les ordres d'Evanturel.

Lundi. Lever à $5 \mathrm{hrs}$. puis parade à 6.30. Dejeuner. Les comp. 3 et 4 se préparent à partir pour Gleichen et les autres places. Ils partent à $11 \mathrm{hrs.} \mathrm{Nous} \mathrm{allons} \mathrm{les} \mathrm{reconduire} \mathrm{aux}$ chars, puis après les discours d'Amyot et d'Evanturel, on se sépare avec regret et peine. Le soir nous jouons au Base-ball et à 8 hrs. séance.

${ }^{26}$ Lieutenant-Colonel Guillaume Amyot, commandant du bataillon. 
Mardi. Lever à $5 \mathrm{hrs}$. Parade. Maintenant on ne fait que de l'extend drill et de l'escarmouching, car le col. nous dit que les affaires de guerre vont très mal pour nous. ${ }^{27}$ et que nous devons nous attendre à nous battre bientôt. La veille j'avais acheté une serviette $35 \mathrm{cts}$ et une livre de beurre $35 \mathrm{cts}$. On devait aller tirer à la cible, mais la partie est remise au lendemain. L'aprèsmidi jusqu'à souper tout le camp est un fatigue party. Je n'ai jamais tant travaillé de la vie et surtout à des affaires aussi malpropres. Je regrettais mon chez nous! Nous eûmes beaucoup de plaisir malgré tout. Le soir revenant de la ville nous parlâmes des affaires de la guerre et les affaires vont très mal pour nous. On parle de nous envoyer en avant. Tout le monde est content. Je me couche...

Fin de ce cahier.

27 En effet les métis avaient repoussé les miliciens du général Middleton à l'Anse-au-Poisson le 24 avril. En même temps les sauvages de Faiseur d'Enclos avaient pillé le village de Battleford et repoussé une expédition du colonel Otter le 2 mai au Couteau-Coupé. 